\title{
SinR is a mutational target for fine-tuning biofilm formation in laboratory-evolved strains of Bacillus subtilis
}

\author{
Sara A Leiman', Laura C Arboleda², Joseph S Spina ${ }^{2,3}$ and Anna L McLoon ${ }^{2,4^{*}}$
}

\begin{abstract}
Background: Bacteria often form multicellular, organized communities known as biofilms, which protect cells from a variety of environmental stresses. During biofilm formation, bacteria secrete a species-specific matrix; in Bacillus subtilis biofilms, the matrix consists of protein polymers and exopolysaccharide. Many domesticated strains of $B$. subtilis have a reduced ability to form biofilms, and we conducted a two-month evolution experiment to test whether laboratory culturing provides selective pressure against biofilm formation in B. subtilis.

Results: Bacteria grown in two-month-long batch culture rapidly diversified their biofilm-forming characteristics, exhibiting highly diverse colony morphologies on LB plates in the initial ten days of culture. Generally, this diversity decreased over time; however, multiple types of colony morphology remained in our final two-month-old populations, both under shaking and static conditions. Notably, while our final populations featured cells that produce less biofilm matrix than did the ancestor, cells overproducing biofilm matrix were present as well. We took a candidate-gene approach to identify mutations in the strains that overproduced matrix and found point mutations in the biofilm-regulatory gene $\sin R$. Introducing these mutations into the ancestral strain phenocopied or partially phenocopied the evolved biofilm phenotypes.

Conclusions: Our data suggest that standard laboratory culturing conditions do not rapidly select against biofilm formation. Although biofilm matrix production is often reduced in domesticated bacterial strains, we found that matrix production may still have a fitness benefit in the laboratory. We suggest that adaptive specialization of biofilm-forming species can occur through mutations that modulate biofilm formation as in B. subtilis.
\end{abstract}

Keywords: Adaptation, Bacteria, Biofilms, Domestication, Laboratory, Selection

\section{Background}

Many species of bacteria form multicellular communities called biofilms, in which aggregated bacterial cells are encased by an extracellular matrix that may comprise polysaccharides, proteins, and nucleic acids [1]. Despite the energetic cost of synthesizing extracellular matrix, cells in biofilms can have a fitness advantage over free-living cells [2]. Biofilms can help cells survive adverse conditions; for example, independent from genetic resistance mechanisms, cells within a biofilm are often more

\footnotetext{
* Correspondence: mcloon@mpi-marburg.mpg.de

${ }^{2}$ Biology Department, Colgate University, Hamilton, NY 13346, USA

${ }^{4}$ Current address: Department of Ecophysiology, MPI for Terrestrial

Microbiology, D-35043 Marburg, Germany

Full list of author information is available at the end of the article
}

resistant to antibiotic treatment than are their planktonic counterparts [3,4]. Biofilms also allow bacteria to form robust communities on both biotic and abiotic surfaces, which can be ecologically beneficial in the environment but which often pose a threat in clinical and industrial settings $[5,6]$. Although biofilm regulatory pathways and the identities of matrix components are species-specific, the advantages of biofilm formation are widespread.

Bacillus subtilis is an endospore-forming bacterium that is frequently found in the soil or associated with plants, and its biofilm-forming abilities have been studied in the laboratory for over a decade $[7,8]$. When $B$. subtilis approaches stationary phase in biofilm-promoting media, the bacteria initially a population of motile single cells and cell chains aggregate and become an ordered biofilm community [9]. 
Within the developing biofilm, a subset of cells secrete a matrix that contains complex polysaccharides, amyloid-like fibers of the protein TasA, and the hydrophobin BslA [10-13]. Notably, the operons responsible for matrix exopolysaccharide (epsA-O) and matrix protein (tapA-sip W-tasA) are under the control of the transcriptional repressor and biofilm master regulator $\operatorname{Sin} R[12,14]$.

In the laboratory environment, $B$. subtilis is often cultured in conditions that do not induce robust biofilm formation, such as constant aeration and the standard laboratory medium LB. Notably, supplementing LB with glycerol and additional manganese can trigger high matrix production in stationary-phase B. subtilis [15]. Given the diversion of resources that occurs during matrix production, we hypothesized that typical culturing conditions might select for B. subtilis mutants that use all of their resources for growth rather than for producing biofilm matrix, even at low levels. Laboratory strains of B. subtilis and of other bacterial species often form less robust biofilms than do their wild ancestors, suggesting that biofilm attenuation is common during domestication [16,17]. Although we believe that historical contingencies (irradiation and repeated transfer from laboratory to laboratory) determined which mutations actually arose in commonly-used laboratory strains of $B$. subtilis such as 168 , we set out to determine whether standard laboratory conditions alone (e.g., rich liquid media, constant aeration) could have selected for the loss of biofilm formation in domesticated B. subtilis.

To examine biofilm formation during extended laboratory culture of $B$. subtilis, we grew multiple independent populations of a robust biofilm-forming strain of $B$. subtilis, NCIB3610 (referred to hereafter as either 3610 or the ancestor). We cultured cells for 60 days, using two different growth conditions, in LB. We regularly saved samples of each evolving population throughout the 60day period, allowing us to monitor colony morphologies over time, and to analyze strains isolated from our final, 60 -day-old populations. To our surprise, we did not uniformly re-domesticate $B$. subtilis, but rather created populations whose members form biofilms with varying levels of robustness on rich medium. Our study suggests that laboratory conditions produce both bacteria with biofilmattenuating mutations and bacteria with biofilm-enhancing mutations. Neither class of mutation fixed in the population over 300 (shaking) or 150 (static) generations. Thus, it is unlikely that standard laboratory culturing alone led to the domestication of biofilm-forming B. subtilis.

\section{Results}

Extended culture produces coexisting cell-types with varied biofilm-forming abilities

Ten independent populations of $B$. subtilis were founded from single colonies as either shaking or static cultures of the robust biofilm-forming strain 3610 and were serially transferred daily or every second day following vigorous vortexing. The cultures were maintained for 60 days, with a calculated average of 5.645 generations per dilution, representing over 338 generations for the shaking culture, and over 169 generations for the static culture. The asynchronicity between the shaking and static cultures was chosen to compensate for the steep oxygen gradient and lower nutrient mixing of static cultures, which disadvantages cell growth. Each of the five shaking and five static populations was sampled regularly and the types and distributions of colony morphologies were examined on LB plates (Figures 1 and 2). Within the first week of culturing, cells forming non-ancestral colony types appeared in all ten cultures, and many of these diverse colony morphologies were maintained at varying proportions for the duration of the experiment (Figure 2).

We classified the colony morphologies of our evolved strains as follows, in order of most to least robust biofilm: irregular wrinkled, wrinkled, wrinkled and fuzzy (FW), fuzzy, ancestral, smooth, or mucoid (Additional file 1: Figure S1). Cells forming these diverse colony morphologies were all visible over the course of the experiment and can be seen in plated samples of the

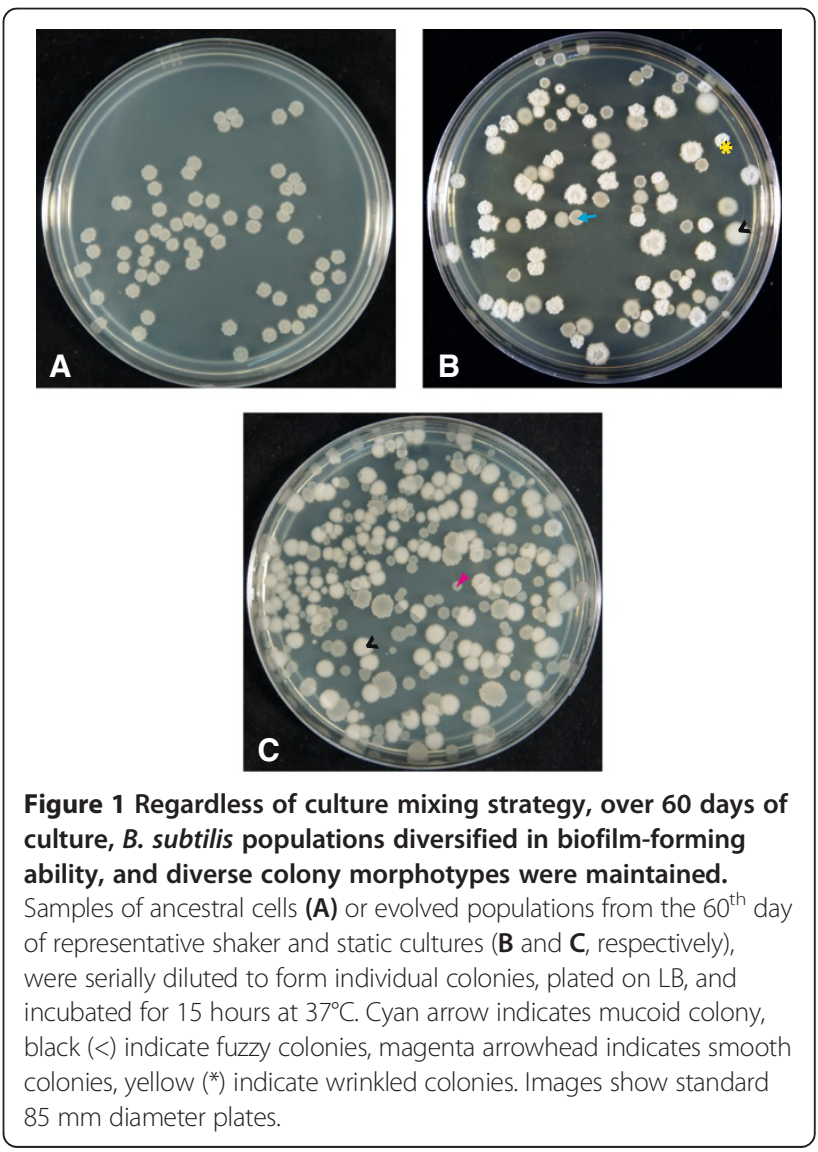



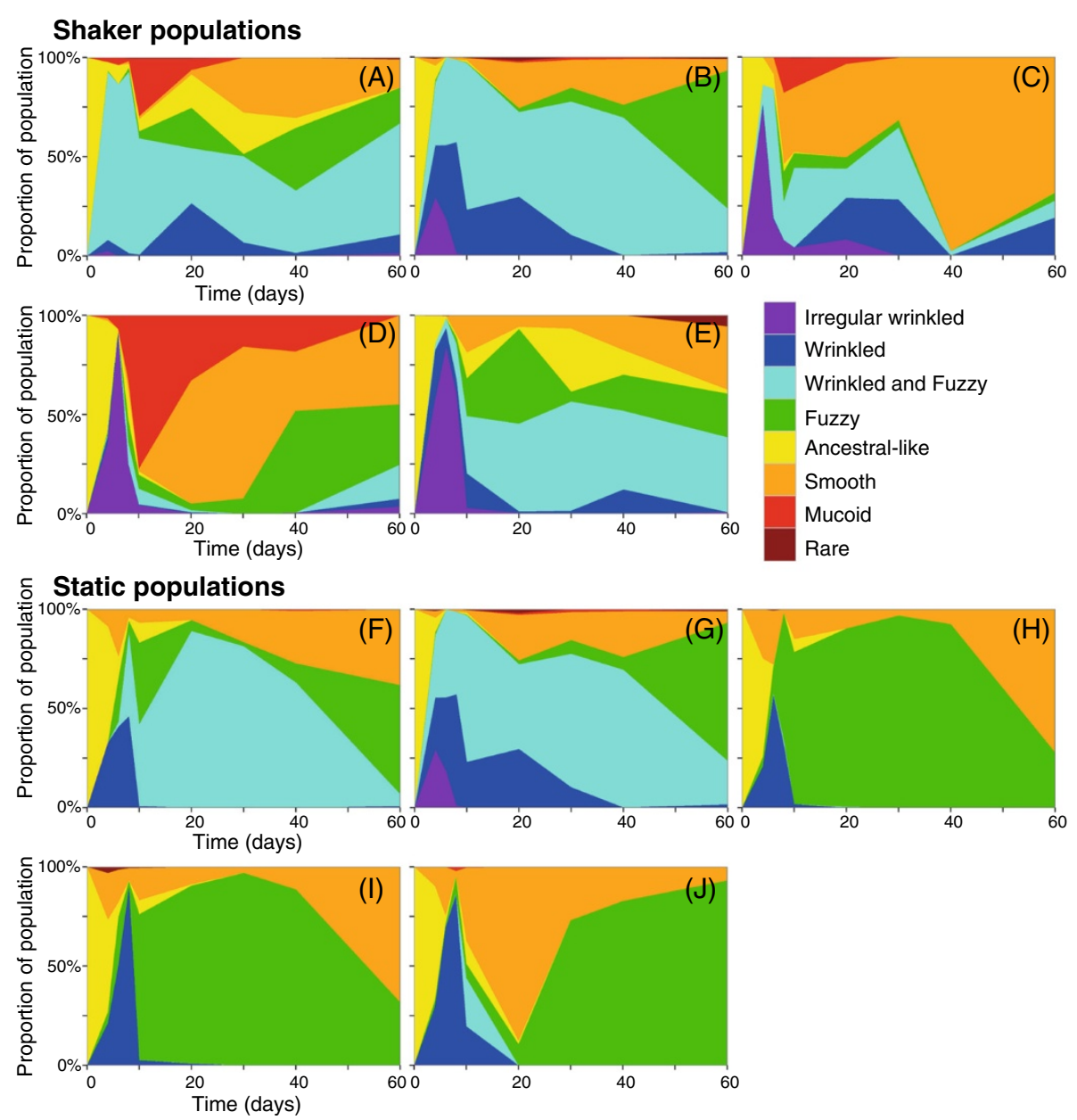

Figure 2 Evolving populations show similar changes in diversity over time. The presence and distribution of colony morphotypes over time were determined for each of the ten two-month extended cultures, using samples from days 4, 6, 8, 10, 20,30, 40, and 60. Shaker populations 1-5 are representated in $\mathbf{A}-\mathbf{E}$, respectively, and static populations 1-5 are represented in $\mathbf{F}-\mathbf{J}$, respectively. The data shown represent the average of two replicates. The colors represent colony morphologies as follows: irregular wrinkled (purple), wrinkled (dark blue), wrinkled and fuzzy (FW, turquoise), fuzzy (green) ancestral-like (yellow), smooth (orange), mucoid (red), and rare morphologies (dark red).

60-day cultures (Figure 2). Cells producing wrinkled, irregular wrinkled or FW colonies rapidly appeared in the shaking cultures, nearly taking over the culture by day 6 or 8 , and then decreasing in abundance over time but never completely disappearing (Figure 2A-E, purple, dark blue and turquoise). Smooth colonyforming cells, on the other hand, appeared in the shaking cultures at later days, and their frequency generally increased over time (Figure 2A, orange). Fuzzy and mucoid colony-forming cells followed less clear patterns in the shaking cultures, becoming prevalent in some cultures but not in others.

Cells forming wrinkled or irregular wrinkled colonies were generally less common in the static cultures than in shaking cultures, whereas cells forming fuzzy or FW colonies were more common (Figure 2F-J). As was the case for the shaking cultures, the prevalence of smooth colony-forming cells in the static cultures increased at later time points. Mucoid colonies were absent from the static cultures apart from static culture 5 , where this morphotype never comprised more than $2 \%$ of the total population.

Following two-month extended culture, we colonypurified 15 colonies from either static or shaking populations. We determined that colony morphology (as observed on LB plates) was a stable trait for the selected isolates. For this study, we then chose to limit further work to a representative sample of five isolates from the day 60 populations that represented the three most distinctive phenotypes: smooth, fuzzy, and wrinkled (Figure 3A, left images). Some strains, including SH1 (shaker 5, day 60, smooth) and SH2 (shaker 3, day 60 , smooth), produced smooth, featureless colonies resembling those made by cells lacking the exopolysaccharide synthesis gene epsH (Figure 3B) [12,18]. Other strains, such as ST1 (static 1, day 60, fuzzy), SH3 (shaker 3, day 


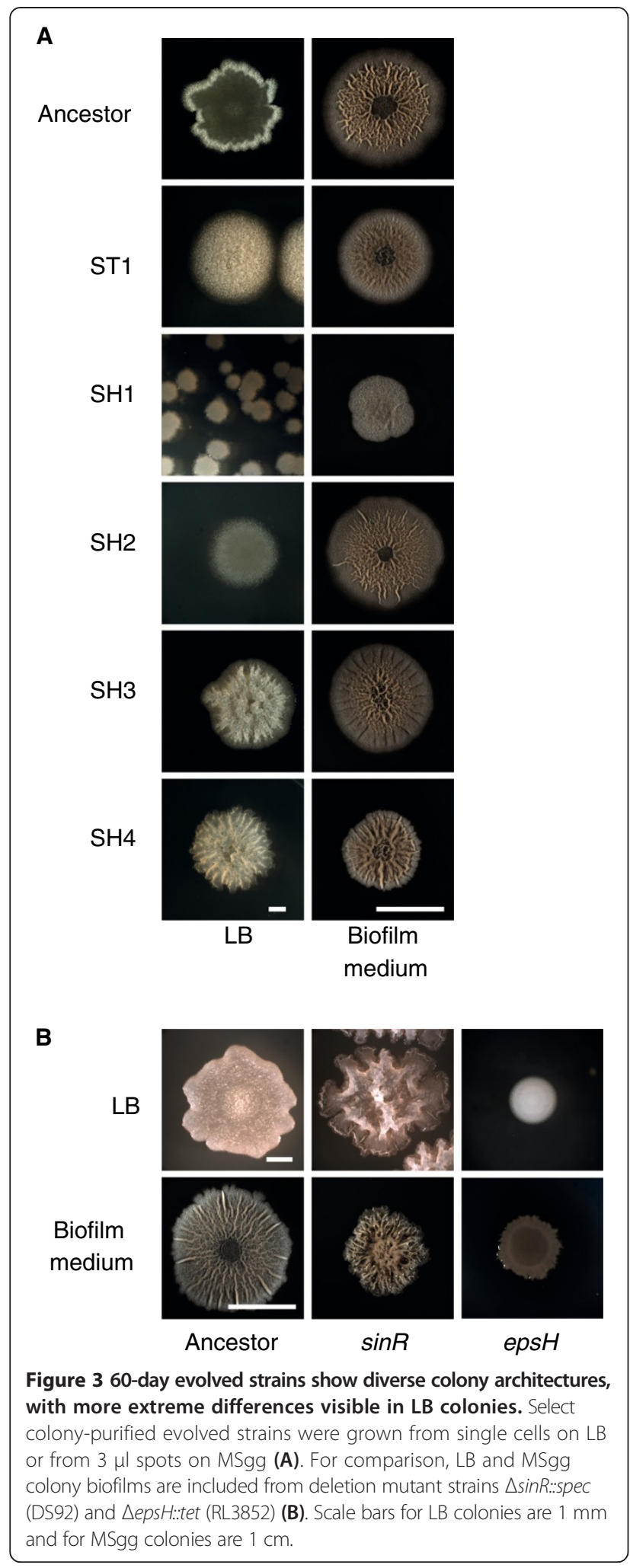

60, wrinkled), and SH4 (shaker 3, day 60, wrinkled), produced colonies with more surface structure than did the ancestral strain (Figure 3A). We hypothesized that the enhanced structure resulted from overproduction of extracellular matrix. Surprisingly, despite their morphological differences on LB, all but one of the isolates characterized here exhibited an ancestral-like, wrinkled colony morphology on the standard nutrient-poor, biofilm-inducing medium MSgg (Figure 3A, right images). This observation suggests that the mutations we recovered during extended culture regulate matrix production in a nutrient-dependent manner, such that matrix gene expression is attenuated or enhanced in nutrient-rich conditions (i.e. LB), but remains the same in nutrient-poor conditions (i.e. MSgg).

\section{Morphology robustness correlates with expression levels of matrix genes}

To test our hypothesis that colony morphology on LB plates corresponds to matrix gene expression in this medium, we constructed luciferase reporters for the operons responsible for the production of matrix exopolysaccharide (epsA-O) and for the production of matrix proteins (tapA-sip $W$-tas $A)$. We measured luminescence for the robust biofilm-forming strains ST1, SH3, and $\mathrm{SH} 4$, as well as the morphologically featureless strain $\mathrm{SH} 2$. Luciferase expression levels for these four evolved strains were compared to those of the ancestral strain and a mutant of the ancestral strain lacking the biofilm repressor gene $\sin R$, which leads to constitutive overexpression of matrix genes [14].

Matrix gene expression - as measured by luminescence - correlated with colony morphology (Figure 4). SH2 did not appear to express matrix genes over basal levels in LB, while ST1, SH3, and SH4 exhibited significantly higher matrix gene expression than that of the ancestral strain (Figure 4). Although $\sin R$ null mutant cells resemble the irregular wrinkled colonies observed early on in several shaking cultures (Figure 3B), none of the fuzzy or wrinkled strains obtained at the experimental endpoint of our extended culture experiment matched the extreme colony morphology or matrix gene expression levels of the $\sin R$ null mutant. These results further support our hypothesis that the mutations we recovered following extended culture resulted in fine-tuning of matrix expression, but caused neither constitutive expression nor, when we additionally consider the MSgg colony phenotypes, a complete absence of biofilm matrix.

\section{Wrinkled colony-forming evolved strains have mutations in the regulatory gene $\sin R$}

Due to the significant role of $\sin R$ in biofilm development, we focused our genetic analysis of our evolved strains on the $\sin R$ locus. SinR tetramers act as transcriptional repressors of matrix genes during vegetative growth, whereas during stationary phase sin R monomers form a complex with either SinI or SlrR. SinI is an antirepressor and can sequester SinR, while SlrR-SinR 


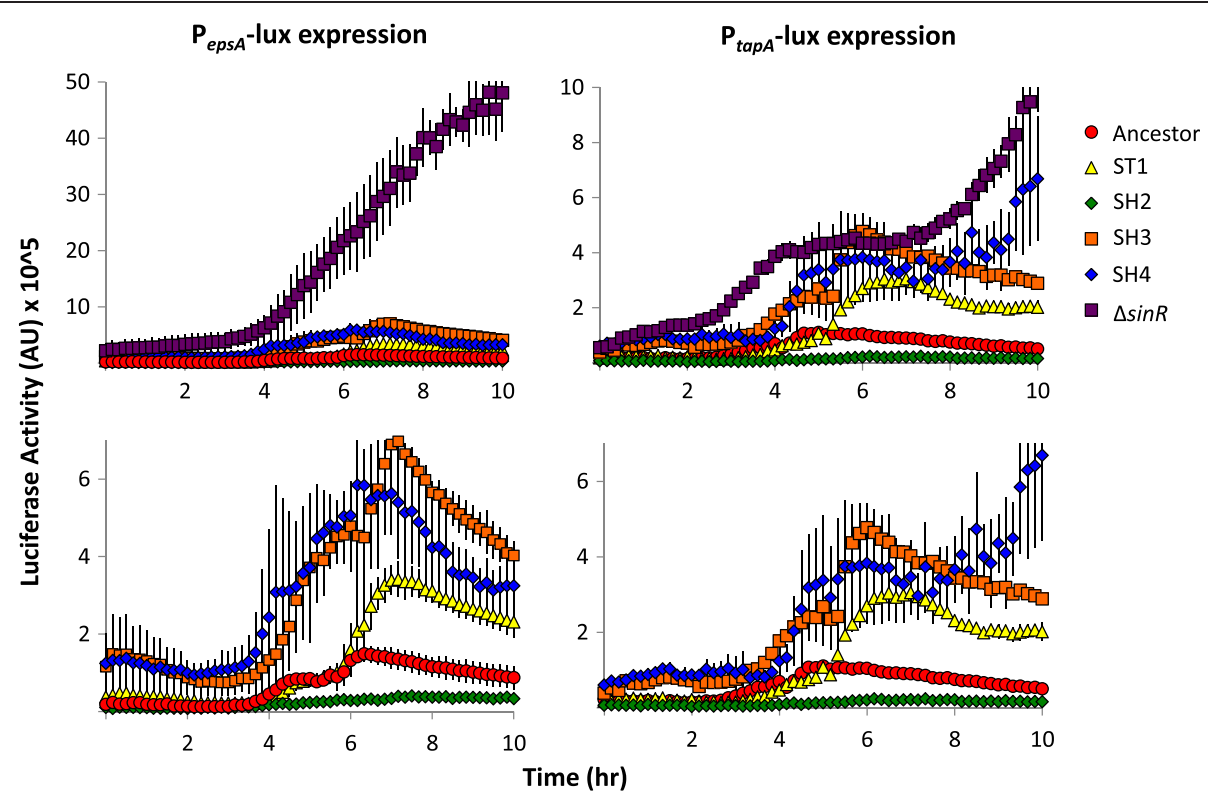

Figure 4 Matrix gene expression of evolved strains corresponds to colony morphology. Select colony-purified evolved strains, the ancestral strain, and a $\sin R$ null mutant of the ancestor were engineered to contain a luciferase reporter under the control of the promoter for either eps $A$ or tapA. All strains were grown and tested in LB. Luciferase activity representative of matrix gene expression was measured every ten minutes and was normalized by culture density. The bottom panels are identical to the top panels but are re-scaled without the $\sin R$ null strain. Results represent the average of at least four replicates and error bars represent the standard deviation.

complexes release repression of the matrix operons and instead repress genes needed for planktonic growth $[14,19,20]$. Sequencing the $\sin R$ locus revealed that strains ST1 and SH3 each carry a mutation in the proteininteraction domain of SinR (Figure 5A), which is the domain responsible for homodimerization and binding with SinI and SlrR. These mutations may modulate SinR binding, by either decreasing SinR dimerization or tetramerization, or possibly by increasing SinR-SlrR or SinI-SinR binding $[21,22]$. A T-to-C point mutation in ST1 changes amino acid 107 from a serine to a proline. In SH3, a G-toA point mutation changes amino acid 89 from a glycine to an arginine. SH4 harbors a silent mutation in $\sin R$ at serine 57 changing the codon from TCG to TCA. Subramaniam et al. showed that serine codon bias within $\sin R$ serves to couple SinR translation to serine levels in the cell [23]. Interestingly, the mutation in SH4 does not fit the general model posed by Subramaniam et al., who observed that mutations changing TCA codons to TCG codons led to higher SinR production and more wrinkled colonies. Instead, we observed the opposite mutation, which nonetheless led to more wrinkled colonies. Regardless, the phenotype of SH4 could be recapitulated by introducing its silent $\sin R$ mutation into a wild-type background. One possible explanation is that this silent serine mutation, which is located $\mathrm{N}$-terminally, could impair the stability of the $\sin R$ transcript (Y. Chai, personal communication).

We note that while the robust biofilm-forming strains ST1, SH3, and SH4 harbored mutations in the master biofilm regulator $\sin R$, the smooth colony-forming strains SH1 and SH2 did not, nor did the smooth isolates have mutations in $\sin I$, the anti-repressor of $\sin R$. Given the presence of $\sin R$ mutations in some of our evolved strains and the fact that SinR is involved in regulating sporulation initiation in addition to biofilm formation, we recognized the possibility that sporulation was also affected in our extended cultures. Thus, we specifically induced and measured sporulation in the evolved isolates and the ancestral strain. Our results did not indicate a correlation between the presence of a mutation in $\sin R$ and sporulation efficiency (Additional file 2: Figure $S 2$ ).

\section{Point mutations in $\sin R$ contribute to the colony morphologies of evolved strains}

Although the identified $\sin R$ mutations seemed likely to be responsible for the observed biofilm phenotypes in the evolved strains, we wanted to rule out a coincidental role for these mutations. To verify that the $\sin R$ mutations in our evolved strains were sufficient to achieve enhanced biofilm robustness, we introduced these specific point mutations into the ancestral strain. Specifically, we reconstituted the $\sin R$ point mutations from ST1, SH3, or $\mathrm{SH} 4$ in the ancestor to create the strains $\mathrm{R} 1\left(\sin R^{319 \mathrm{~T}>\mathrm{C}}\right)$, $\mathrm{R} 3\left(\sin R^{265 \mathrm{G}>\mathrm{A}}\right)$, andR4 $\left(\sin R^{171 \mathrm{G}>\mathrm{A}}\right)$, respectively. As demonstrated by colony phenotype on LB agar, each of the reconstituted strains exhibits significant matrix overexpression when compared to the ancestor (Figure 5B). R3 


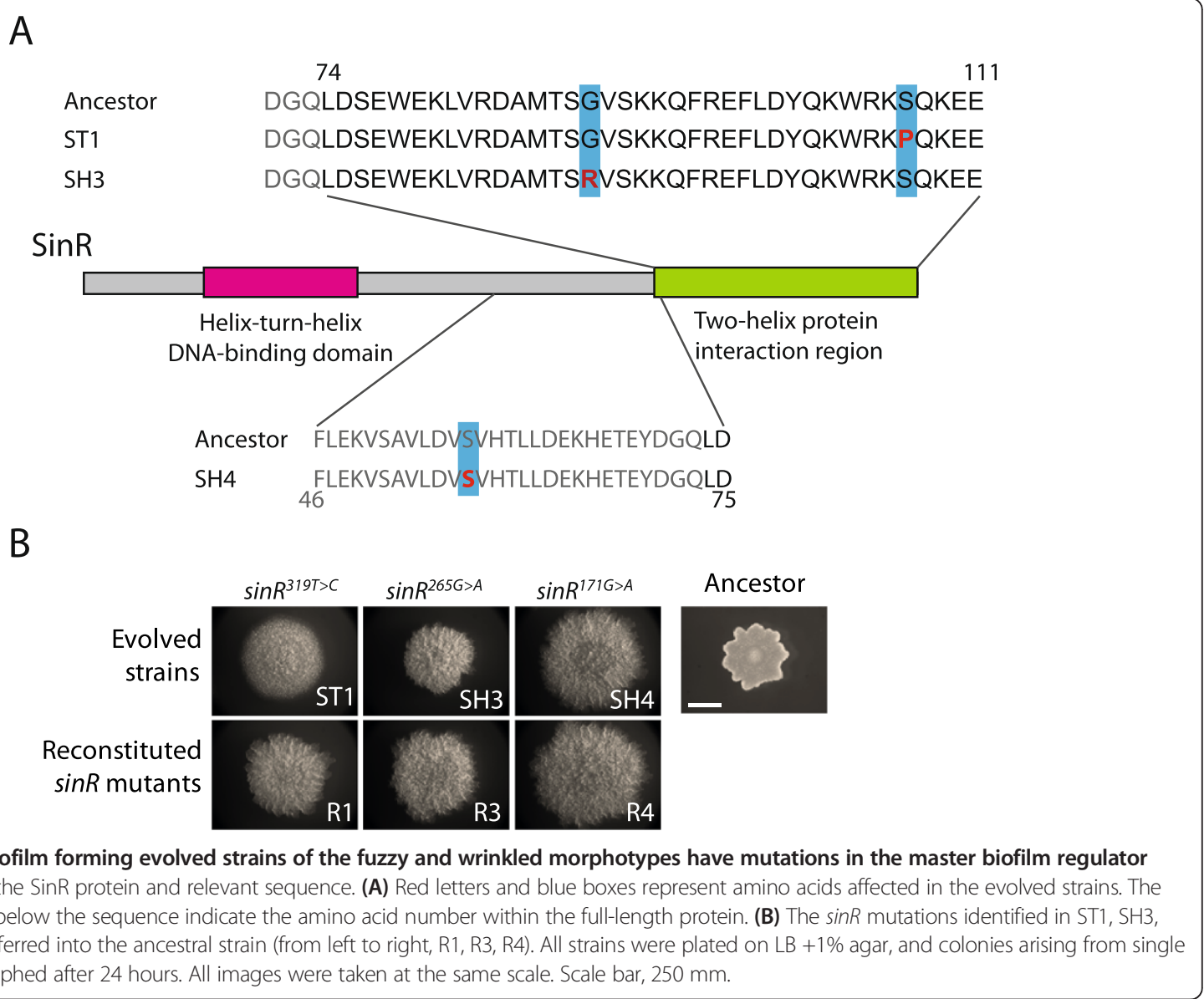

and R4 closely phenocopied $\mathrm{SH} 3$ and SH4, respectively, indicating that these $\sin R$ mutations alone may be responsible for the evolved colony morphologies. R1 partially phenocopied the fuzzy colony-producing strain ST1 (Figure 5B). R1 had more pronounced biofilm structure than did ST1, suggesting that ST1 harbors at least one other mutation that tempers the phenotype caused by the $\sin R$ mutation. Overall, we found that point mutations in $\sin R$ arose readily during extended culture of $B$. subtilis, that these mutations are sufficient for robust biofilm formation in wrinkled colony types, and that these mutations can contribute to fuzzy colony types as well.

\section{Discussion}

We conducted a two-month long-term evolution experiment to determine how extended laboratory culture changes biofilm formation in B. subtilis. Although laboratory-domesticated strains of $B$. subtilis do not form robust biofilms, two months of culture of the wild ancestor resulted in the emergence of strains with a diversity of biofilm robustness, as demonstrated by colony phenotype and the expression of matrix-producing genes. While the appearance and prevalence of strain types in each evolving culture varied over time and among replicate populations, none of our culture conditions reproducibly led to uniform attenuation or loss of biofilm formation. Instead, many of the evolved strains formed more robust biofilms than the ancestral strain and these appeared early in our populations and were thereafter present in high numbers. This suggests that matrix overproduction can be neutral, if not advantageous, even in rich medium.

We attempted to test the fitness of our evolved matrixoverproducing strains in competition with the ancestral strain and with the smooth colony variants. Unfortunately, the matrix-overproducing strains form aggregates, making these experiments highly variable and impracticable to interpret (data not shown). The same issue presented in our attempts to perform growth curves using spectrophotometric or plating methods, as the aggregates of matrixoverexpressing strains did not disperse, even following vigorous vortexing. Nonetheless, with the abundance of fuzzy and/or wrinkled colony-forming cells frequently in excess of $90 \%$ of the population, we feel confident that these cells have a strong selective advantage during 60 days of culture.

The high proportion and persistence of fuzzy colonyproducing cells in particular may indicate that a 
moderate rise in biofilm matrix production grants cells the largest fitness advantage. Perhaps these cells are able to adhere to and colonize niches, such as the wall of a tube, unavailable to cells that make lower levels of matrix. Similarly, it is plausible that fuzzy colony-forming cells have greater fitness over robustly wrinkled colony-forming cells, because the former can allocate more resources toward proliferation.

The diversity of smooth, fuzzy, and wrinkled colonies that we observed after extended culture is reminiscent of the phenotypes observed by Rainey and Travisano in spatially structured cultures of Pseudomonas fluorescens [24]. Their work also led to fuzzy and wrinkled colony types, and similarly, their wrinkled phenotypes were due to mutations leading to overproduction of a polymer matrix [25]. Such results suggest that hyper-production of matrix is a common adaptation that allows bacteria to utilize the ecological niche of a culture's air-medium or vessel-air-medium interface.

Finally, some strains in our final populations exhibited reduced colony complexity and partly resembled domesticated laboratory strains, and these became more prevalent towards the end of our experiment. It is possible that over longer culturing, they might win out due to clonal interference or drift, even though non-matrixforming strains did not increase to fixation within our populations during the observed generations. We hope to explore the genetic bases for the observed biofilm attenuation in future work. The production of matrix is energetically costly, but is clearly not without benefits, even in standard laboratory conditions.

\section{Conclusions}

Although biofilm formation has well-accepted advantages under many "real-world" conditions, the attenuation of biofilm formation in some laboratory bacterial strains raises questions of the fitness effects of biofilm formation under laboratory conditions. Here, we demonstrate that biofilm formation can still be advantageous in the laboratory for B. subtilis, and that moderate changes in matrix production may allow bacteria to colonize specific niches even in seemingly homogeneous cultures. Further, we identified several specific mutations in one key matrix regulatory gene, $\sin R$, which led to increased production of matrix in subsets of our evolved populations. Because biofilm formation evolves rapidly and frequently during laboratory culture, our results suggest that biofilm-deficient laboratory strains of $B$. subtilis were selected by scientists rather than by culture conditions alone.

\section{Methods}

\section{Strains and culture conditions}

The strains used in this study are listed in Table 1. Strains were routinely grown in LB medium (10 g/liter tryptone, $5 \mathrm{~g} /$ liter yeast extract, $10 \mathrm{~g} /$ liter $\mathrm{NaCl}$ ), TY medium (LB supplemented with $10 \mathrm{mM} \mathrm{MgSO}_{4}$ and $100 \mu \mathrm{M} \mathrm{MnSO}_{4}$ ), MSgg medium (5 $\mathrm{mM}$ potassium phosphate, $100 \mathrm{mM}$ morpholinepropanesulfonic acid [MOPS] pH 7, $2 \mathrm{mM} \mathrm{MgCl} 2,50 \mu \mathrm{M} \mathrm{MnCl}_{2}, 50 \mu \mathrm{M}$ $\mathrm{FeCl}_{3}, 700 \mu \mathrm{M} \mathrm{CaCl}, 1 \mu \mathrm{M} \mathrm{ZnCl}, 2 \mu \mathrm{M}$ thiamine, $0.5 \%$ glycerol, $0.5 \%$ glutamate, $50 \mu \mathrm{g} / \mathrm{ml}$ threonine, tryptophan, and phenylalanine), or Difco Sporulation medium [26]. Colonies initiated from single cells were grown in LB for 15 hours at $37^{\circ} \mathrm{C}$ from serially diluted starter cultures. MSgg colony biofilms were inoculated with $3 \mu \mathrm{l}$ of starter culture, allowed to dry, and incubated for 3 days at $30^{\circ} \mathrm{C}$. As appropriate, antibiotics were added at the following concentrations: tetracycline $(10 \mu \mathrm{g} / \mathrm{ml})$, chloramphenicol $(5 \mu \mathrm{g} / \mathrm{ml})$, kanamycin $(5 \mu \mathrm{g} / \mathrm{ml})$, X-Gal (5-bromo-4-chloro3-indolyl- $\beta$-D-galactopyranoside; $100 \mu \mathrm{g} / \mathrm{ml}), 1 \mu \mathrm{g} / \mathrm{ml}$ erythromycin, and $25 \mu \mathrm{g} / \mathrm{ml}$ lincomycin.

\section{Strain construction}

Promoters to the epsA and tapA operons were PCR amplified from genomic DNA using 5'-TGGCGAATTCTGTACGGCTTGCACTAAATGTAC-3' and 5'-GTTCGTC GACATTCATAGCCTTCAGCCTTCCCG-3' (epsA), and 5'-GTTCGTCGACATCTTACCTCCTGTAAAACACTG-3' and 5'-TGGCGAATTCATAGACAAATCACACATTG TTTG-3' (tapA) primers, cloned into plasmid pAH321 and transferred into domesticated B. subtilis strain PY79 as previously described $[27,28]$. Reporter constructs and marker-linked mutations or gene knockouts were moved into the appropriate strains by transduction with the Spp1 bacteriophage following previously described methods [29].

Mutated $\sin R$ was amplified from the evolved isolates using the following primers: 5'-CGTTGTAAAACGAC GGCCAGTGAATTCGTCTTCACCTAGTCTCTGGAAC-3' and 5 '-AACAGCTATGACCATGATTACGCCAAGCT TCATTCAATAAAAGGGGAGCTTACC-3'. Mutations in $\sin R$ were markerlessly reconstructed in B. subtilis 3610 using the pMiniMAD protocol as described by Patrick and Kearns [30]. Successful double-crossover events were indicated by the absence of growth in the presence of erythromycin and lincomycin. Mutations in $\sin R$ were subsequently verified using 5'-TGGATCAAGAATGGG TTGAATTAATGGT-3' and 5'-CAGCGCCATTAGAGA AATTGAAAGAAAG-3'.

\section{Extended batch culture}

Five replicate $5 \mathrm{ml} \mathrm{LB}$ cultures in glass test tubes were inoculated with single colonies of NCIB3610 and incubated at $37^{\circ} \mathrm{C}$ in an orbital shaker (150 rpm). Every 24 hours, for 60 days, the cultures were vortexed for 30 seconds, then $100 \mu \mathrm{l}$ of the shaking cultures were transferred to fresh $5 \mathrm{~mL}$ LB cultures. This transfer scheme represented $10^{6}-10^{8}$ cells transferred, and we hoped would minimize inadvertent bottleneck events or 
Table 1 Bacterial strains used in this work

\begin{tabular}{|c|c|c|}
\hline Strain name & Genotype & Source or strain construction \\
\hline $\mathrm{NCIB3610}$ & ancestral biofilm-forming strain & Laboratory stock \\
\hline PY79 & domesticated laboratory strain & Laboratory stock \\
\hline 168 & domesticated laboratory strain & Laboratory stock \\
\hline DS92 & $\Delta \sin R::$ spec & Kearns et al. [14] \\
\hline RL3852 & $\triangle e p s H:: t e t$ & Kearns et al. [14] \\
\hline R1 & $\sin R^{319 T>C}$ in NCIB3610 & This study \\
\hline R3 & $\sin R^{265 \mathrm{G}>\mathrm{A}}$ in $\mathrm{NCIB} 3610$ & This study \\
\hline R4 & $\sin R^{171 G>A}$ in $\mathrm{NCIB} 3610$ & This study \\
\hline ST1 & evolved strain isolated from static culture 1 on day 60 & This study \\
\hline $\mathrm{SH} 1$ & evolved strain isolated from shaking culture 5 on day 60 & This study \\
\hline $\mathrm{SH} 2$ & evolved strain isolated from shaking culture 3 on day 60 & This study \\
\hline $\mathrm{SH} 3$ & evolved strain isolated from shaking culture 3 on day 60 & This study \\
\hline $\mathrm{SH} 4$ & evolved strain isolated from shaking culture 3 on day 60 & This study \\
\hline ALM89 & sacA::P epsA-lux in NCIB3610, $\mathrm{Cm}^{\mathrm{R}}$ & This study \\
\hline SLH2O & sacA:: $P_{\text {epsA }}-$ lux in ST1, $\mathrm{Cm}^{\mathrm{R}}$ & This study \\
\hline $\mathrm{SLH} 21$ & sacA::P $P_{e p s}-l u x$ in $\mathrm{SH} 2, \mathrm{Cm}^{\mathrm{R}}$ & This study \\
\hline SLH22 & sacA::P $P_{\text {eps }}-\mathrm{lux}$ in $\mathrm{SH} 3, \mathrm{Cm}^{\mathrm{R}}$ & This study \\
\hline $\mathrm{SLH} 23$ & sacA:: $P_{\text {eps }}-\mathrm{lux}$ in $\mathrm{SH} 4, \mathrm{Cm}^{\mathrm{R}}$ & This study \\
\hline $\mathrm{SLH} 24$ & sacA::P $P_{\text {epsA }}-$ lux in DS92, $\mathrm{Cm}^{\mathrm{R}}$ & This study \\
\hline ALM91 & sacA::P tapA-lux in NCIB3610, $\mathrm{Cm}^{\mathrm{R}}$ & This study \\
\hline $\mathrm{SLH} 25$ & sacA:: $P_{\text {tapA }}-$ lux in ST1, $\mathrm{Cm}^{R}$ & This study \\
\hline SLH26 & sacA:: $P_{\text {tapA }}-\mathrm{lux}$ in $\mathrm{SH} 2, \mathrm{Cm}^{\mathrm{R}}$ & This study \\
\hline SLH27 & sacA:: $P_{\text {tapA }}-1 \mathrm{ux}$ in $\mathrm{SH} 3, \mathrm{Cm}^{\mathrm{R}}$ & This study \\
\hline $\mathrm{SLH} 28$ & sacA::P $P_{\text {tapA }}-$ lux in $\mathrm{SH} 4, \mathrm{Cm}^{\mathrm{R}}$ & This study \\
\hline $\mathrm{SLH} 29$ & sacA: $: P_{\text {tapA }}-/$ ux in DS92, $\mathrm{Cm}^{\mathrm{R}}$ & This study \\
\hline
\end{tabular}

the accidental exclusion of clumpy cell types that would be underrepresented in smaller volumes. Alternatively, five replicate $5 \mathrm{ml} \mathrm{LB}$ cultures in glass test tubes were inoculated with single colonies of NCIB3610 and incubated statically at $37^{\circ} \mathrm{C}$. Every 48 hours, the cultures were vigorously vortexed for 30 seconds and $100 \mu$ l were transferred to fresh LB cultures, for 60 total days. Every other day for the first 3 weeks and every week thereafter, samples from each culture were taken and stored at $-80^{\circ} \mathrm{C}$ in $20 \%$ glycerol. Cultures were also regularly tested for contamination by plating serial dilutions of the evolving populations.

\section{Quantification of colony morphologies over time}

Two samples of each population from days 4, 6, 8, 10, $20,30,40$, and 60 were grown from glycerol stocks for 3 hours at $37^{\circ} \mathrm{C}$ in $3 \mathrm{~mL}$ LB. The samples were then serially diluted and plated on LB agar plates, incubated for 15 hours at $37^{\circ} \mathrm{C}$, and imaged by digital photography. All colonies on each plate were counted, with a minimum of 50 colonies and an average of 150-200 colonies counted per sample. The resulting colonies were counted and classified by morphology and the relative abundances of each morphology were averaged and graphed using the $\mathrm{R}$ package ggplot2 [31].

\section{Sequencing}

$\sin R$ was sequenced using the following primers for amplification and sequencing 5' ${ }^{\prime}$-TGGATCAAGAATGG GTTGAATTAATGGT-3' and 5'-CAGCGCCATTAGA GAAATTGAAAGAAAG-3'.

\section{Kinetic luciferase assay}

Cells were grown in LB to mid-log phase and diluted 1:100 in fresh LB. Dilutions were plated in quadruplet (250 $\mu \mathrm{l}$ each) in a 96-well polystyrene Costar plate (white with a clear bottom; Fisher Scientific, USA). The luciferase activity of each strain was measured on a BioTek Synergy 2 luminometer (BioTek, USA) with continuous slow shaking at $30^{\circ} \mathrm{C}$. Luciferase luminescence was measured at a sensitivity setting of 200 , and culture optical density was measured at $600 \mathrm{~nm}$ every 10 minutes for 
24 hours. Final luciferase activity values were calculated by normalizing luciferase luminescence to culture density. Data shown represents the average of at least four biological replicates.

\section{Spore counts}

Heat-resistant spores of the ancestral and selected evolved strains were produced, isolated and counted as previously described [26]. Spores for each strain were counted in triplicate and the entire experiment was replicated three times.

\section{Additional files}

Additional file 1: Figure S1. Representative colonies for each class of colony morphology. Images represent individual colonies from the plates featured in Figure 2. The colony diameter is in part affected by the colony density on the plates. Colony morphologies are as follows: irregular wrinkled $(A, B)$, wrinkled (C-E), wrinkled and fuzzy (FW) (F), fuzzy $(G)$ ancestral-like $(H)$, smooth $(I, J)$, and mucoid (K,L). Scale bar, $500 \mathrm{~mm}$

Additional file 2: Figure S2. Spore counts for the ancestor and select evolved strains. The indicated strains were grown in liquid DSM for 28 hours, after which heat-resistant spores were isolated and plated. The resulting colonies were counted in triplicate for each strain. The data shown represent the average of three independent experiments (normalized to the ancestor for each experiment) and the error bars represent the standard deviation.

\section{Competing interests}

The authors declare that they have no competing interests.

\section{Authors' contributions}

SAL participated in strain characterization and $\sin R$ sequencing, conducted kinetic luciferase assays, constructed strains, and helped draft the manuscript. LCA and JSS participated in two-month extended culture, selection of representative evolved strains, and initial characterization of these strains. ALM conceived the study, participated in its design and coordination, participated in two-month extended culture, strain characterization, and $\sin R$ sequencing, and helped draft the manuscript. All authors read and approved the final manuscript.

\section{Acknowledgements}

Thanks to M. Rowley for help propagating and maintaining the two-month extended cultures, to A. Keyel for help with ggplot2 implementation, and to K. Belanger, R. Losick, and S. Mango for reagents and workspace. This work was supported by a discretionary grant from the Colgate Research Council (to ALM). SAL is supported by grant GM18568 (to R. Losick) and ALM is supported by a long-term fellowship from the Alexander von Humboldt Foundation.

\section{Author details}

'Department of Molecular and Cellular Biology, Harvard University, Cambridge, MA 02138, USA. ${ }^{2}$ Biology Department, Colgate University, Hamilton, NY 13346, USA. ${ }^{3}$ Current address: Channing Division of Network Medicine, Brigham and Women's Hospital and Harvard Medical School, Boston, MA 02115, USA. ${ }^{4}$ Current address: Department of Ecophysiology, MPI for Terrestrial Microbiology, D-35043 Marburg, Germany.

Received: 17 June 2014 Accepted: 18 November 2014

Published online: 30 November 2014

\section{References}

1. Flemming HC, Wingender J: The biofilm matrix. Nat Rev Microbio/ 2010, 8:623-633.

2. Jefferson KK: What drives bacteria to produce a biofilm? FEMS Microbiol Lett 2004, 236:163-173.
3. Mah TF, O'Toole GA: Mechanisms of biofilm resistance to antimicrobial agents. Trends Microbiol 2001, 9:34-39.

4. Hill D, Rose B, Pajkos A, Robinson M, Bye P, Bell S, Elkins M, Thompson B, MacLeod C, Aaron SD, Harbour C: Antibiotic susceptibilities of Pseudomonas aeruginosa isolates derived from patients with cystic fibrosis under aerobic, anaerobic, and biofilm conditions. J Clin Microbiol 2005, 43:5085-5090.

5. Costerton JW, Stewart PS, Greenberg EP: Bacterial biofilms: a common cause of persistent infections. Science 1999, 284:1318-1322.

6. Davey ME, OToole GA: Microbial biofilms: from ecology to molecular genetics. Microbiol Mol Biol Rev 2000, 64:847-867.

7. Branda SS, Gonzalez-Pastor JE, Ben-Yehuda S, Losick R, Kolter R: Fruiting body formation by Bacillus subtilis. Proc Natl Acad Sci U S A 2001, 98:11621-11626.

8. Hamon MA, Lazazzera BA: The sporulation transcription factor Spo0A is required for biofilm development in Bacillus subtilis. Mol Microbiol 2001, 42:1199-1209.

9. Kobayashi K: Bacillus subtilis pellicle formation proceeds through genetically defined morphological changes. J Bacterio/ 2007, 189:4920-4931.

10. Romero D, Aguilar C, Losick R, Kolter R: Amyloid fibers provide structural integrity to Bacillus subtilis biofilms. Proc Natl Acad Sci U S A 2011, 107:2230-2234.

11. Romero $D$, Vlamakis $H$, Losick $R$, Kolter $R$ : An accessory protein required for anchoring and assembly of amyloid fibres in B. subtilis biofilms. Mol Microbiol 2010, 80:1155-1168.

12. Branda SS, Chu F, Kearns DB, Losick R, Kolter R: A major protein component of the Bacillus subtilis biofilm matrix. Mol Microbio/ 2006, 59:1229-1238

13. Hobley L, Ostrowski A, Rao FV, Bromley KM, Porter M, Prescott AR, MacPhee CE, van Aalten DM, Stanley-Wall NR: BsIA is a self-assembling bacterial hydrophobin that coats the Bacillus subtilis biofilm. Proc Natl Acad Sci US A 2013, 110:13600-13605.

14. Kearns DB, Chu F, Branda SS, Kolter R, Losick R: A master regulator for biofilm formation by Bacillus subtilis. Mol Microbiol 2005, 55:739-749.

15. Shemesh $M$, Chai $Y$ : A combination of glycerol and manganese promotes biofilm formation in Bacillus subtilis via histidine kinase KinD signaling. J Bacteriol 2013, 195:2747-2754.

16. Aguilar C, Vlamakis H, Losick R, Kolter R: Thinking about Bacillus subtilis as a multicellular organism. Curr Opin Microbiol 2007, 10:638-643.

17. McLoon AL, Guttenplan SB, Kearns DB, Kolter R, Losick R: Tracing the domestication of a biofilm-forming bacterium. J Bacterio/ 2011, 193:2027-2034.

18. Branda SS, Gonzalez-Pastor JE, Dervyn E, Ehrlich SD, Losick R, Kolter R: Genes involved in formation of structured multicellular communities by Bacillus subtilis. J Bacteriol 2004, 186:3970-3979.

19. Chai $Y$, Kolter R, Losick R: Paralogous antirepressors acting on the master regulator for biofilm formation in Bacillus subtilis. Mol Microbiol 2009, 74:876-887.

20. Chai Y, Norman T, Kolter R, Losick R: An epigenetic switch governing daughter cell separation in Bacillus subtilis. Genes Dev 2010, 24:754-765.

21. Colledge VL, Fogg MJ, Levdikov VM, Leech A, Dodson EJ, Wilkinson A Structure and organisation of $\operatorname{SinR}$, the master regulator of biofilm formation in Bacillus subtilis. J Mol Biol 2011 411:597-613.

22. Newman JA, Rodrigues C, Lewis RJ: Molecular basis of the activity of SinR protein, the master regulator of biofilm formation in Bacillus subtilis. J Biol Chem 2013, 288:10766-10778.

23. Subramaniam AR, Deloughery A, Bradshaw N, Chen Y, O'Shea E, Losick R, Cha $Y$ : A serine sensor for multicellularity in a bacterium. elife 2013, 2:e01501.

24. Rainey PB, Travisano M: Adaptive radiation in a heterogeneous environment. Nature 1998, 394:69-72.

25. Bantinaki E, Kassen R, Knight CG, Robinson Z, Spiers AJ, Rainey PB: Adaptive divergence in experimental populations of Pseudomonas fluorescens. III. Mutational origins of wrinkly spreader diversity. Genetics 2007, 176:441-453.

26. Schaeffer $P$, Millet J, Aubert JP: Catabolic repression of bacterial sporulation. Proc Natl Acad Sci U S A 1965, 54:704-711.

27. McLoon AL, Kolodkin-Gal I, Rubinstein SM, Kolter R, Losick R. Spatia regulation of histidine kinases governing biofilm formation in Bacillus subtilis. J Bacterio/ 2011, 193:679-685.

28. Schmalisch M, Maiques E, Nikolov L, Camp AH, Chevreux B, Muffler A, Rodriguez S, Perkins J, Losick R: Small genes under sporulation control in the Bacillus subtilis genome. J Bacteriol 2010, 192:5402-5412. 
29. Yasbin RE, Young FE: Transduction in Bacillus subtilis by bacteriophage SPP1. J Virol 1974, 14:1343-1348.

30. Patrick JE, Kearns DB: MinJ (YvjD) is a topological determinant of cell division in Bacillus subtilis. Mol Microbiol 2008, 70:1166-1179.

31. Wickham H: ggplot2: Elegant Graphics for Data Analysis. Berlin: Springer; 2009.

doi:10.1186/s12866-014-0301-8

Cite this article as: Leiman et al: SinR is a mutational target for fine-

tuning biofilm formation in laboratory-evolved strains of Bacillus subtilis. BMC Microbiology 2014 14:301.

\section{Submit your next manuscript to BioMed Central and take full advantage of:}

- Convenient online submission

- Thorough peer review

- No space constraints or color figure charges

- Immediate publication on acceptance

- Inclusion in PubMed, CAS, Scopus and Google Scholar

- Research which is freely available for redistribution 